\title{
LA-UR-21-26873
}

Approved for public release; distribution is unlimited.

Title: $\quad$ Secure System Composition and Type Checking using Cryptographic Proofs

Author(s): $\quad$ Barrack, Daniel Abraham

Intended for: $\quad$ For discussion with other ZKSnark researchers

Issued: 
Disclaimer:

Los Alamos National Laboratory, an affirmative action/equal opportunity employer, is operated by Triad National Security, LLC for the National Nuclear Security Administration of U.S. Department of Energy under contract 89233218CNA000001. By approving this article, the publisher recognizes that the U.S. Government retains nonexclusive, royalty-free license to publish or reproduce the published form of this contribution, or to allow others to do so, for U.S. Government purposes. Los Alamos National Laboratory requests that the publisher identify this article as work performed under the auspices of the U.S. Department of Energy. Los Alamos National Laboratory strongly supports academic freedom and a researcher's right to publish; as an institution, however, the Laboratory does not endorse the viewpoint of a publication or guarantee its technical correctness. 


\section{Secure System Composition and Type Checking using Cryptographic Proofs}

\section{Dani Barrack}

A-4: Advanced Research in Cyber Systems

Email: dbarrack@lanl.gov

Mentor: Michael J. Dixon

Co-Mentor: Boris Gelfand

(1) Information Science

June 21st, 2021

D \& Technology Institute

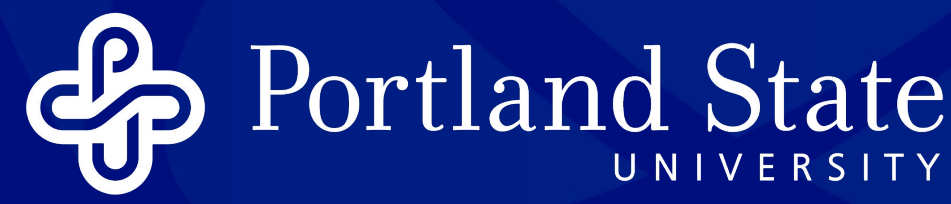




\section{Challenge: Formally Verifying System Composition}

We can use formal methods to verify that systems compose correctly without the possibility of incorrect behavior.

This means exhaustively checking that System A's postconditions agree with System B's preconditions. If so, it is safe to compose.

Normal Setting: Every computational path must be accounted for and checked. Verification cost (time) is multiplicative across systems.

$$
\operatorname{Cost}=\left|\mathrm{S}_{1}\right| *\left|\mathrm{~S}_{2}\right| * \ldots *\left|\mathrm{~S}_{\mathrm{n}}\right|
$$

System A:

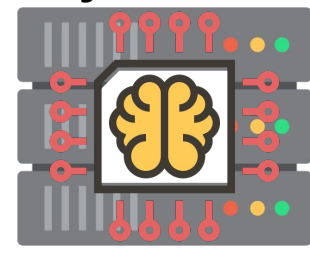

Train ML Model

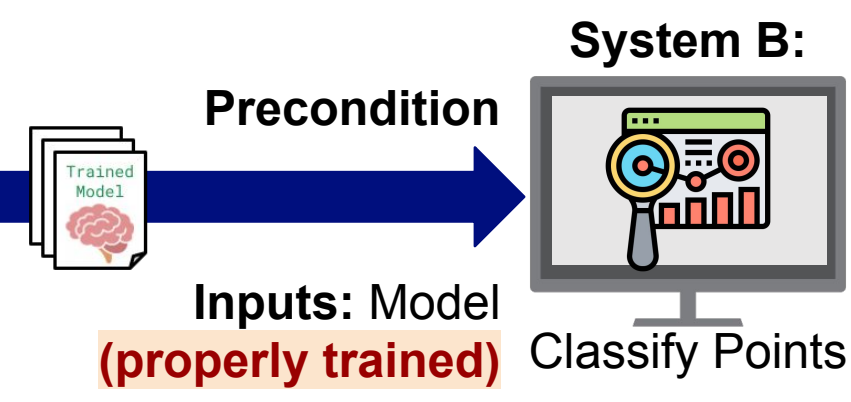

Outputs: Model
Postcondition

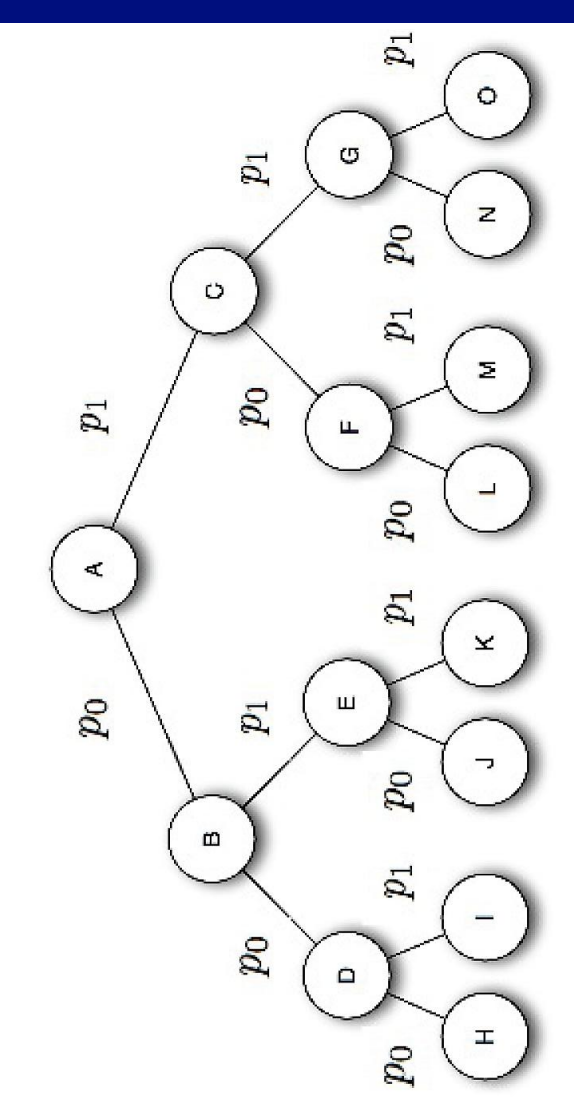




\section{Solution: Assuring Safe Composition via zkSNARKs}

Zero-knowledge proofs can be used to provide type checking guarantees of input/output properties without exposing secrets.

Verification can be done modularly so that the cost is additive.

$$
\text { Cost }=\left|\mathrm{S}_{1}\right|+\left|\mathrm{S}_{2}\right|+\ldots+\left|\mathrm{S}_{\mathrm{n}}\right|
$$

Bad proofs and inputs can still exist, but now are cryptographically (exponentially) hard to find and exploit.

Postcondition

System A:

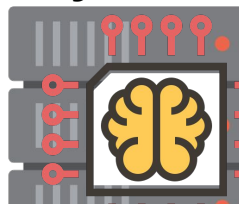

Train ML Model
Outputs: Trained model
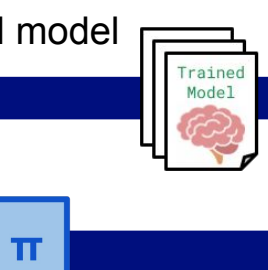

zkSNARK proving model is valid
Precondition Inputs: Trained model System B:

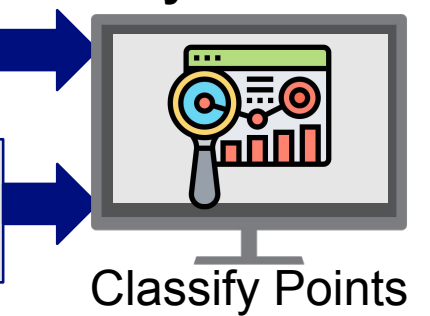

ZKP Verifier
Classify Points

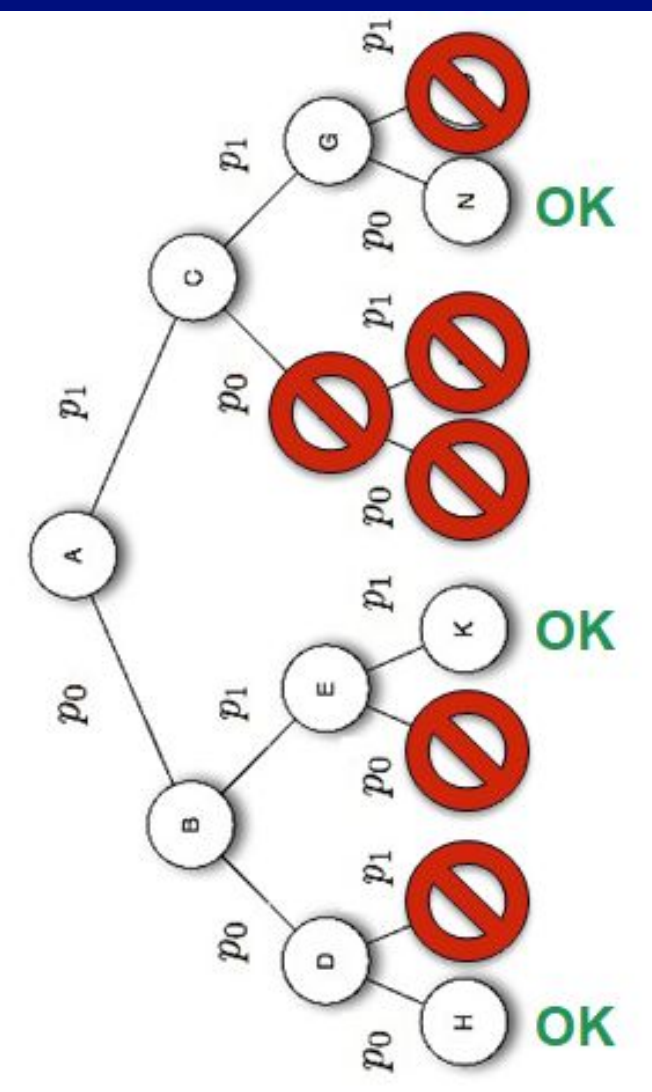




\section{Preconditions and Postconditions with Types}

Type MLModel $=$

$(w$ : Weights, error $(w)<0.05, \log :$ AuditLog, execute $(\log )==w)$

trainModel : (x : [ Input] ) $\rightarrow$ MLModel

classifyPoint : (y : Input, model : MLModel) $\rightarrow$ Class

We can generate proofs (or an audit log) of desired properties (e.g. functional correctness) and include them with input to other functions.

This allows us to use a dependent type to assure that only models that were actually trained on actual data and are within a certain error threshold can be used by a classifier.

The audit log and its proof would be huge. Instead, we can use a super small zkSNARK to prove this dependent type and pass it along instead. We only need to handle the case where the check fails. 


\section{Preconditions and Postconditions with Types}

Type MLModel = zkp : ZKP

(w : Weights,

trainModel : (x: [ Input] $) \rightarrow$ MLModel

classifyPoint : (y : Input, model : MLModel, verif : ZKPVerifier $\rightarrow$ I0 Class

We can generate proofs (or an audit log) of desired properties (e.g. functional correctness) and include them with input to other functions.

This allows us to use a dependent type to assure that only models that were actually trained on actual data and are within a certain error threshold can be used by a classifier.

The audit log and its proof would be huge. Instead, we can use a super small zkSNARK to prove this dependent type and pass it along instead. We only need to handle the case where the check fails. 


\section{Dependent Type Replacement by ZKPs}

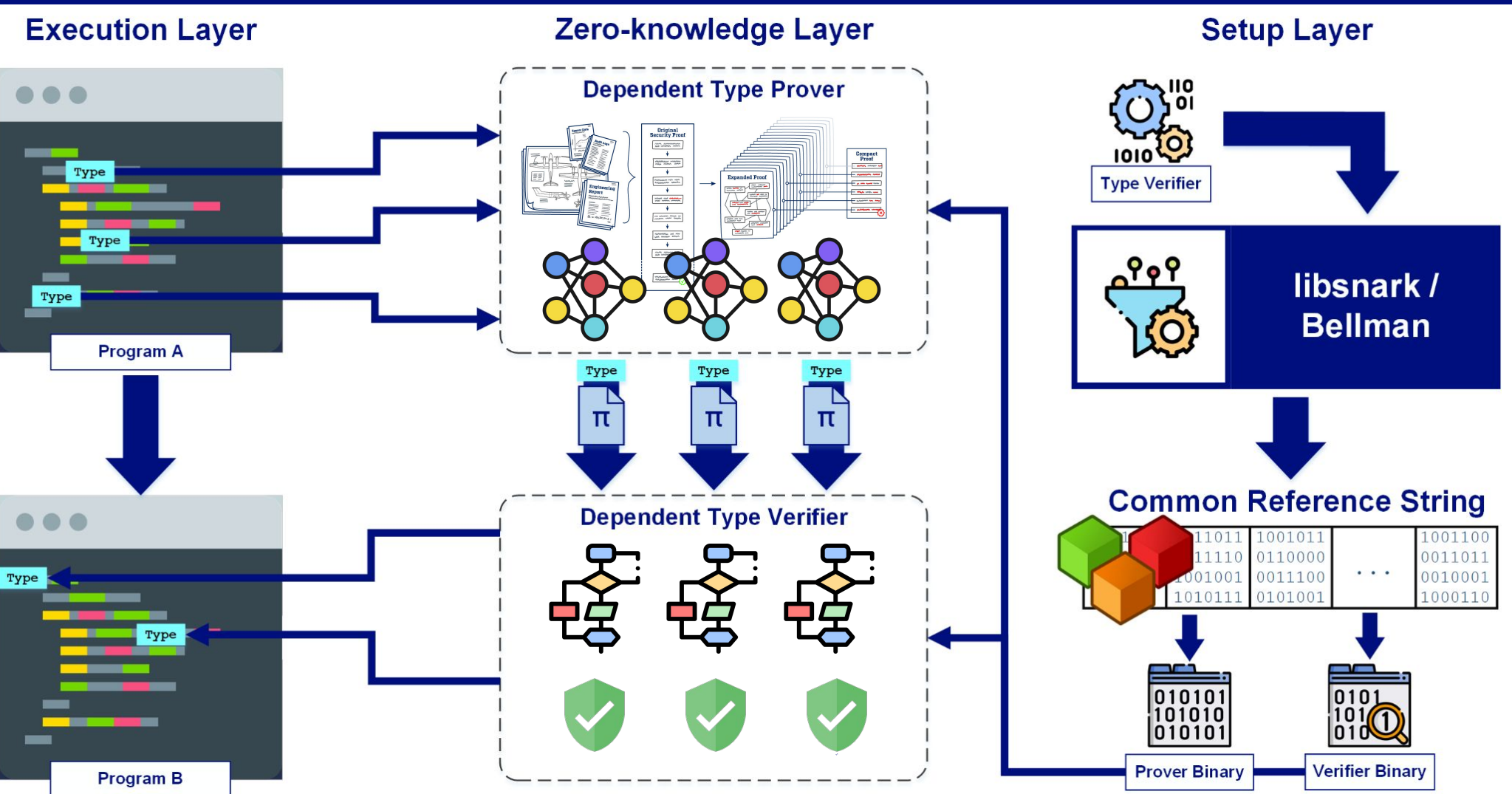




\section{Benefit \& Capability}

Using zero-knowledge proofs we can combine systems while preventing certain incorrect behaviors relating to mismatched outputs and input constraints.

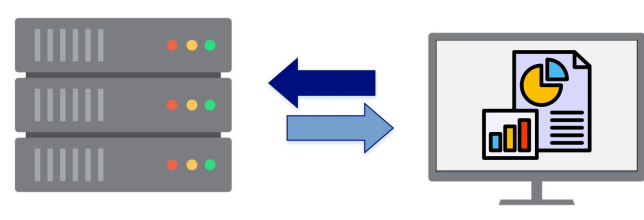

ZKPs enforce system compatibility without the expense of manually proving correctness

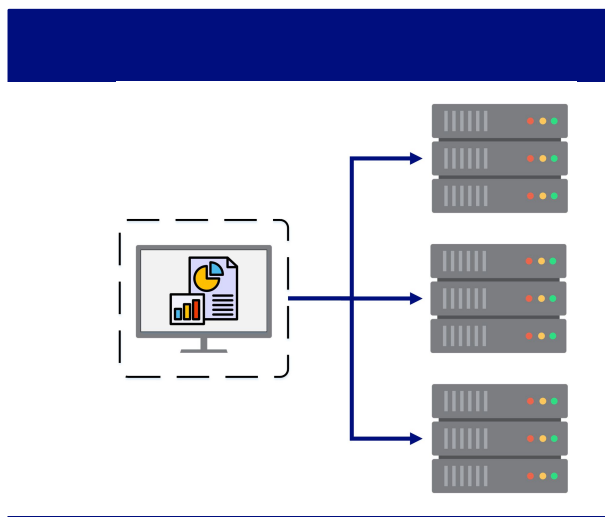

Portable proofs artificially extends our trusted computing base beyond just our own system

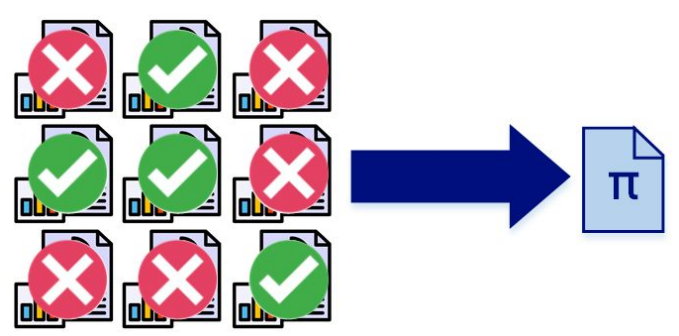

ZKPs give fine-grained control over which bits of information to keep secret and which to prove 


\section{Project Roadmap}

1. Prototype and test program interoperability

- Manually implement skeleton code in place of zero-knowledge proofs to interact with example program

2. Implement constraint related ZKP gadgets

- Constraints capture type information that is immediately useful to the test program

3. Develop and set up prototype demonstrations

- Replace skeleton code with handcrafted ZKP gadgets

- Develop prototype compiler to read type annotations from file and generate constraints

4. Benchmark and Evaluate 


\section{Backup}


Our function as a circuit

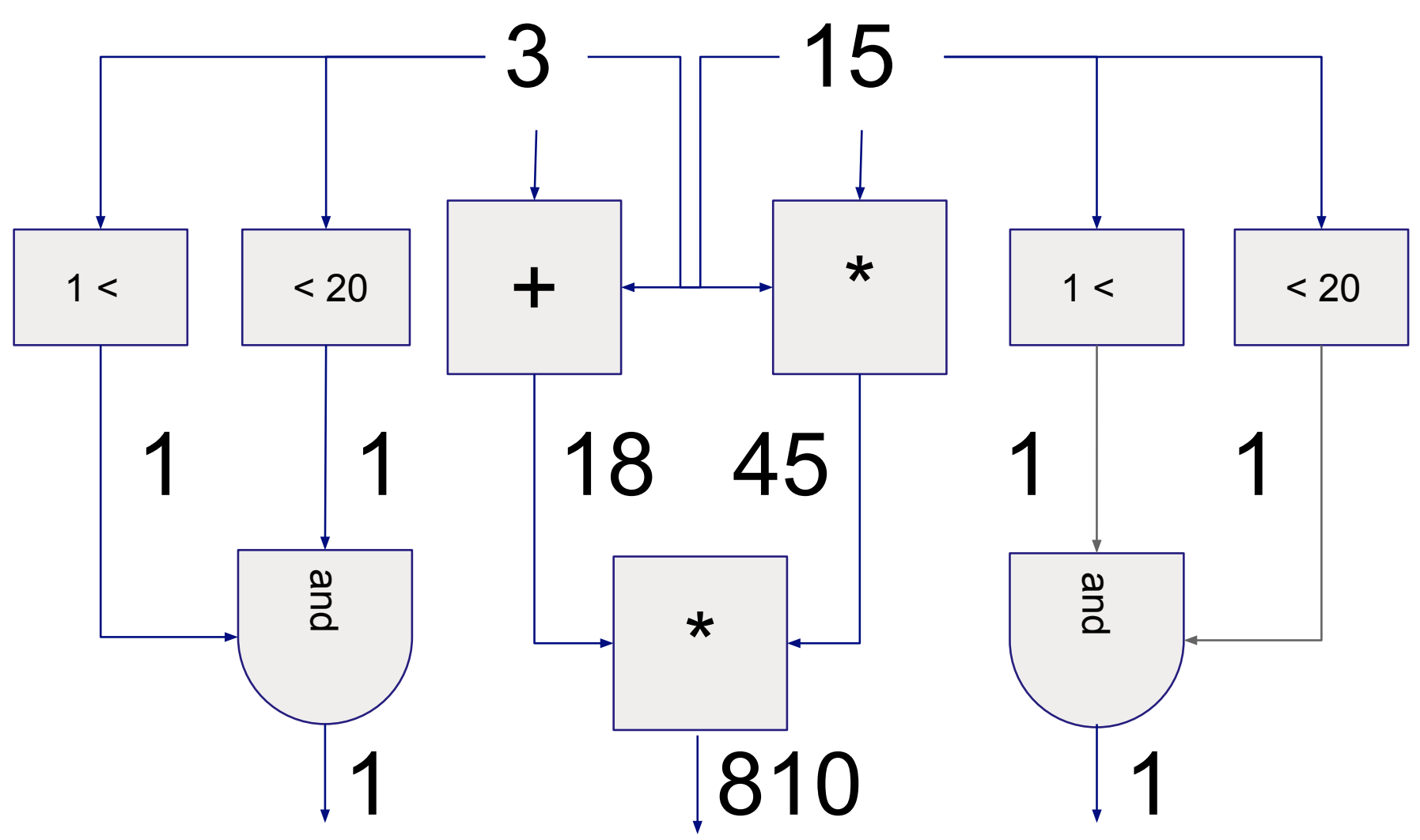


A redacted circuit with zkSNARKs

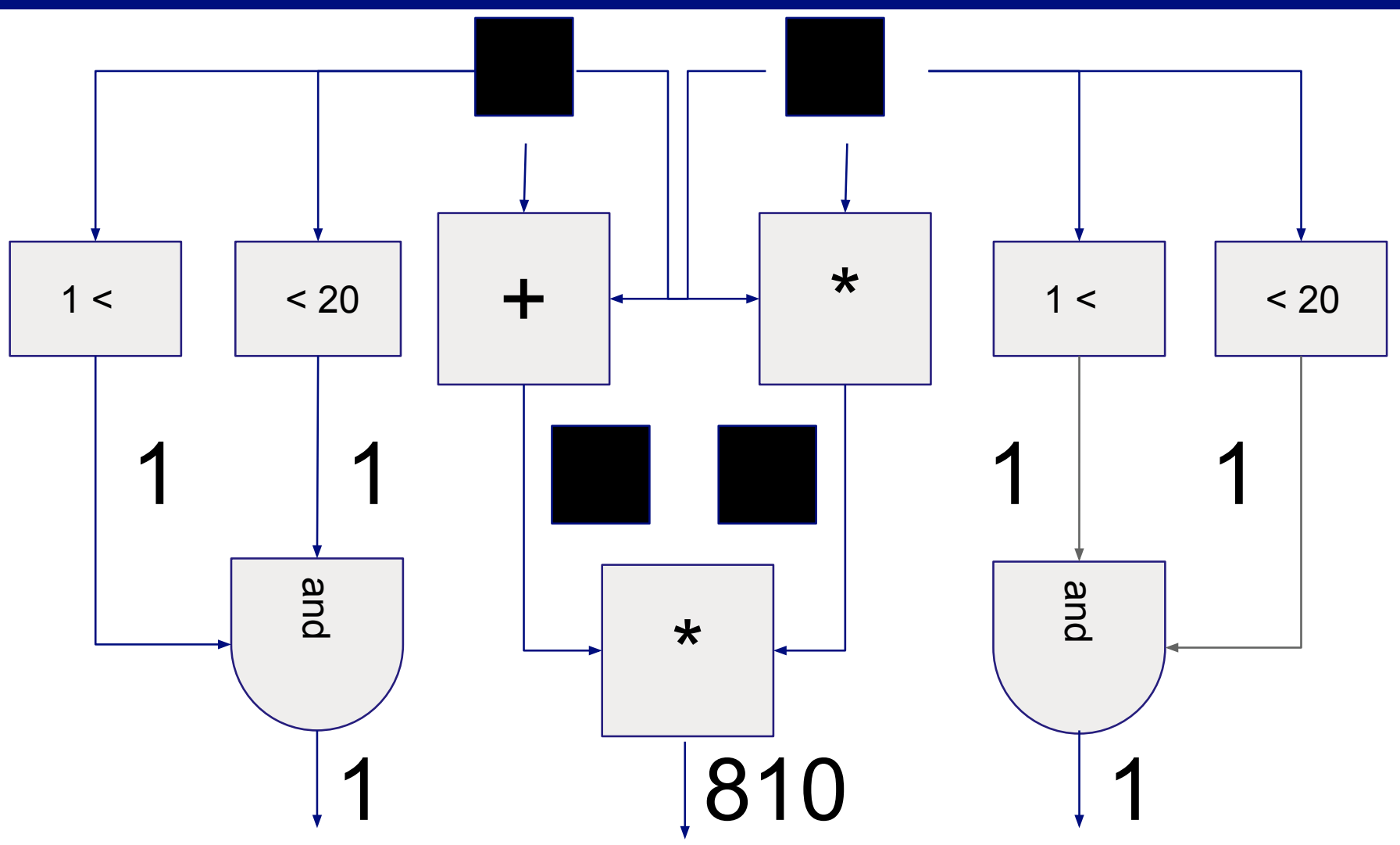


A redacted circuit with zkSNARKs

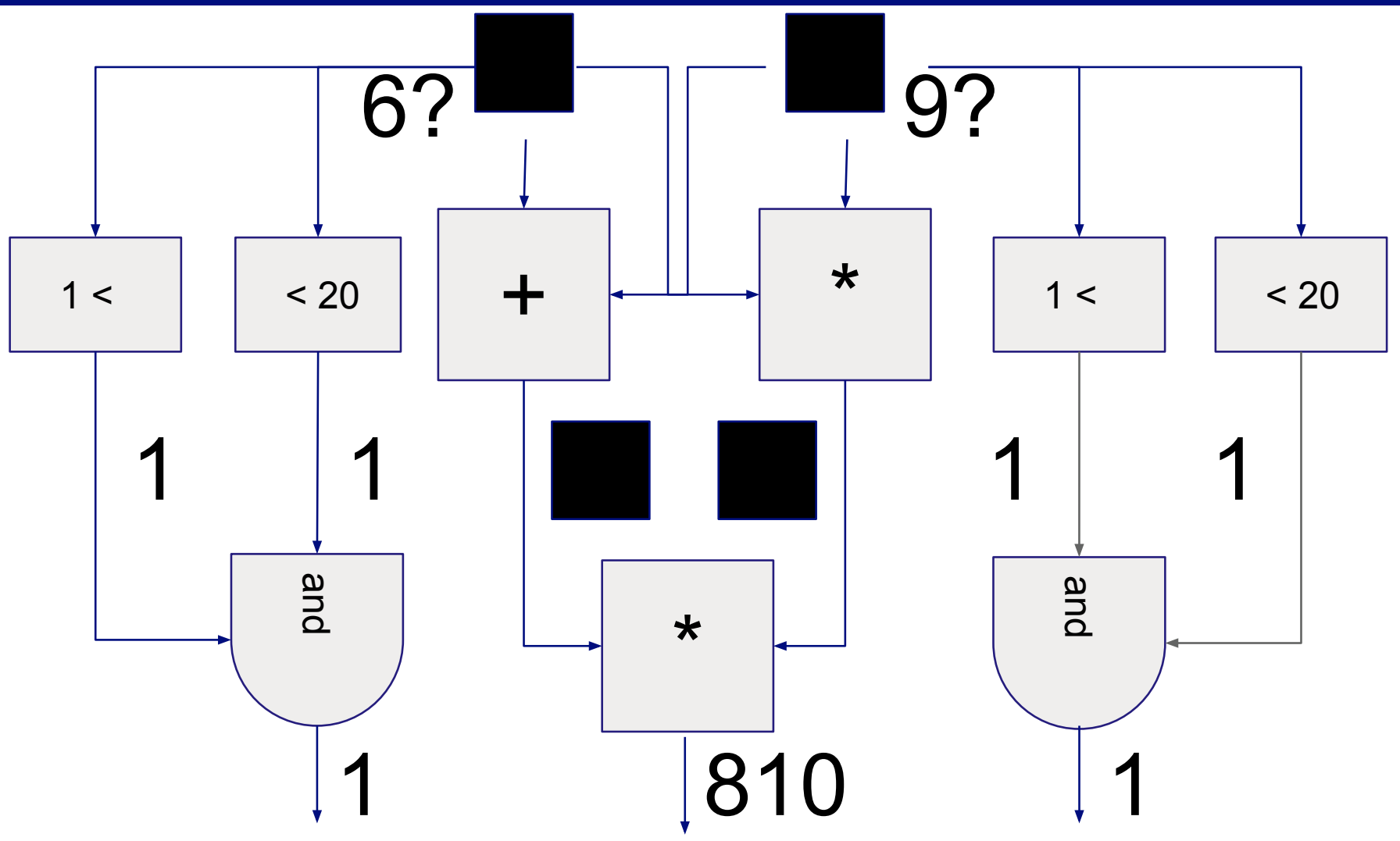




\section{Zero-Knowledge Proof for Where's Waldo?}

Example. You want to prove that you have beaten Where's Waldo?

- Traditional Proof: Point to Waldo to demonstrate you know where he is

- Not zero-knowledge!

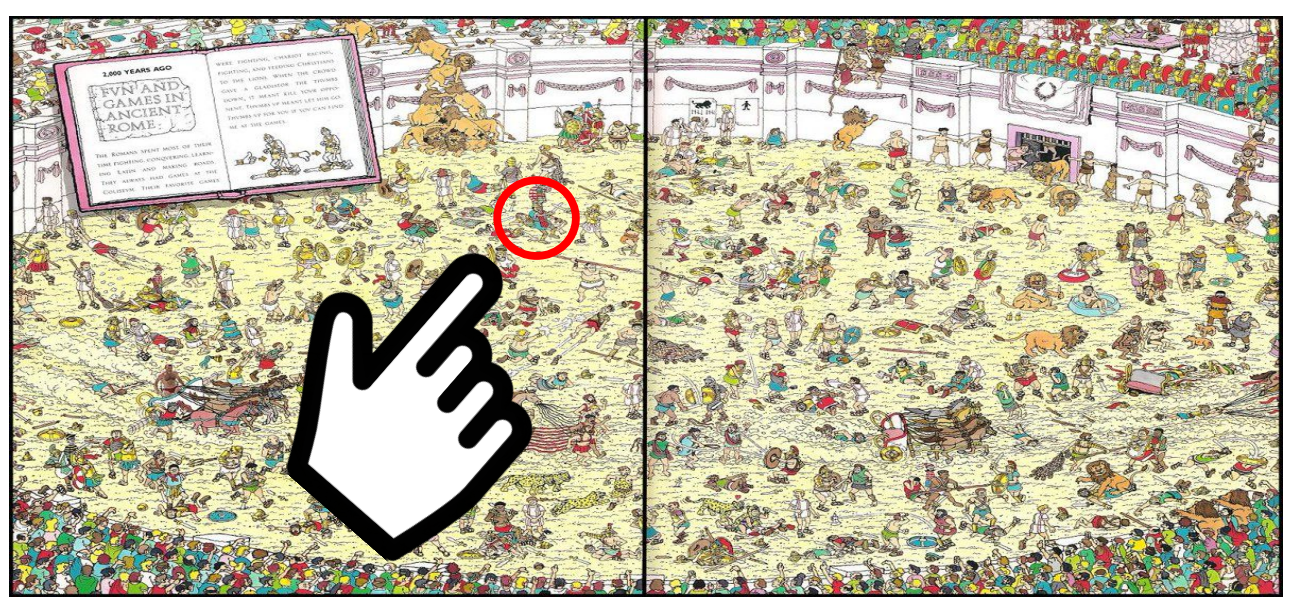

This kind of proof leaks all information about his location, much more than simply that you have knowledge of the location 


\section{Zero-Knowledge Proof for Where's Waldo?}

Zero-knowledge proof for "Where's Waldo?"

1. Cut out a Waldo shaped hole in a much larger piece of paper

2. Position the hole over Waldo's location

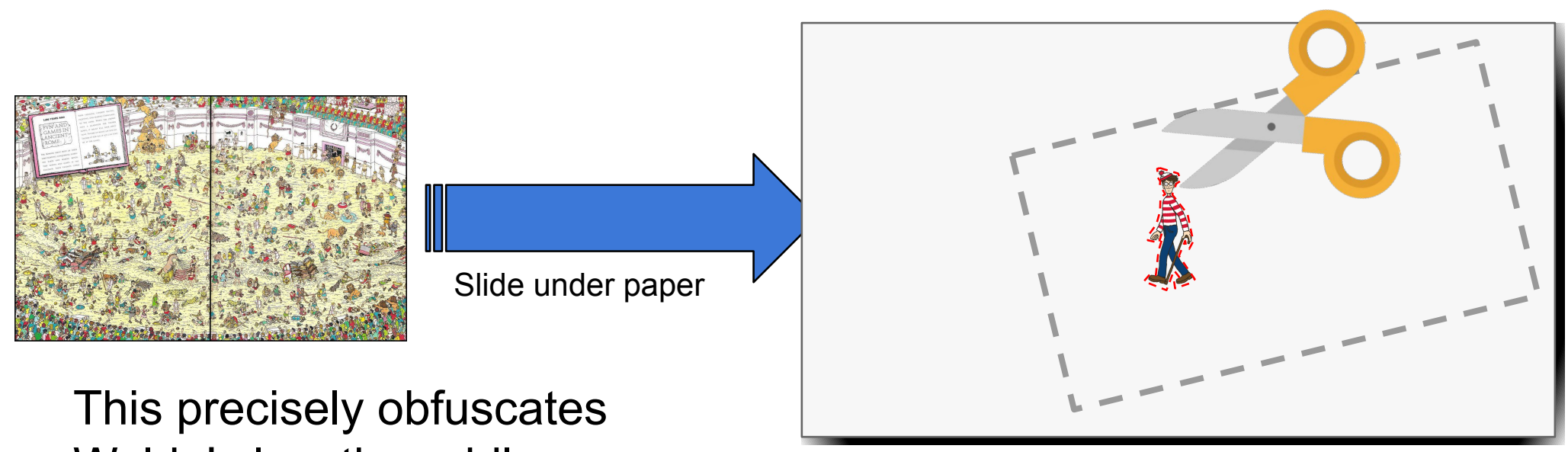

Waldo's location while demonstrating knowledge of his whereabouts!

To adversaries, the book underneath could hypothetically be in any random orientation 


\section{Completeness vs. Soundness}

Typical proof systems have $100 \%$ completeness and $100 \%$ soundness

Completeness: $\quad$ P $[$ true statement AND verifier accepts] $=1$ "Everything true is provable"

Soundness:

P[false statement AND verifier rejects] $=1$

"False statements aren't provable" 


\section{Cryptographic Proof Systems}

Cryptographic proof systems have variable completeness and soundness. For non-interactive zero-knowledge proofs we care about:

(Completeness) $\quad$ P [true statement AND verifier accepts $]=1$ "Everything true is provable"

(Soundness)

$P$ [false statement AND verifier rejects] $=1-\varepsilon$ "Low chance that a proof of a false statement is encountered"

We sacrifice minimal amount of soundness (have to break crypto to produce counter-example) in order to get valuable proof properties 


\section{Zero-Knowledge Proofs and Verifiable Computation}

Zero-knowledge proofs (ZKPs) allow us to prove that a claim IS true without revealing WHY it is true, even if the prover is untrusted and malicious.

zkSNARKs are special ZKPs that are tiny and non-interactive

Inputs:

Logs

Schematics

Program Traces

Signals

Encryption Keys

Attestations

etc.

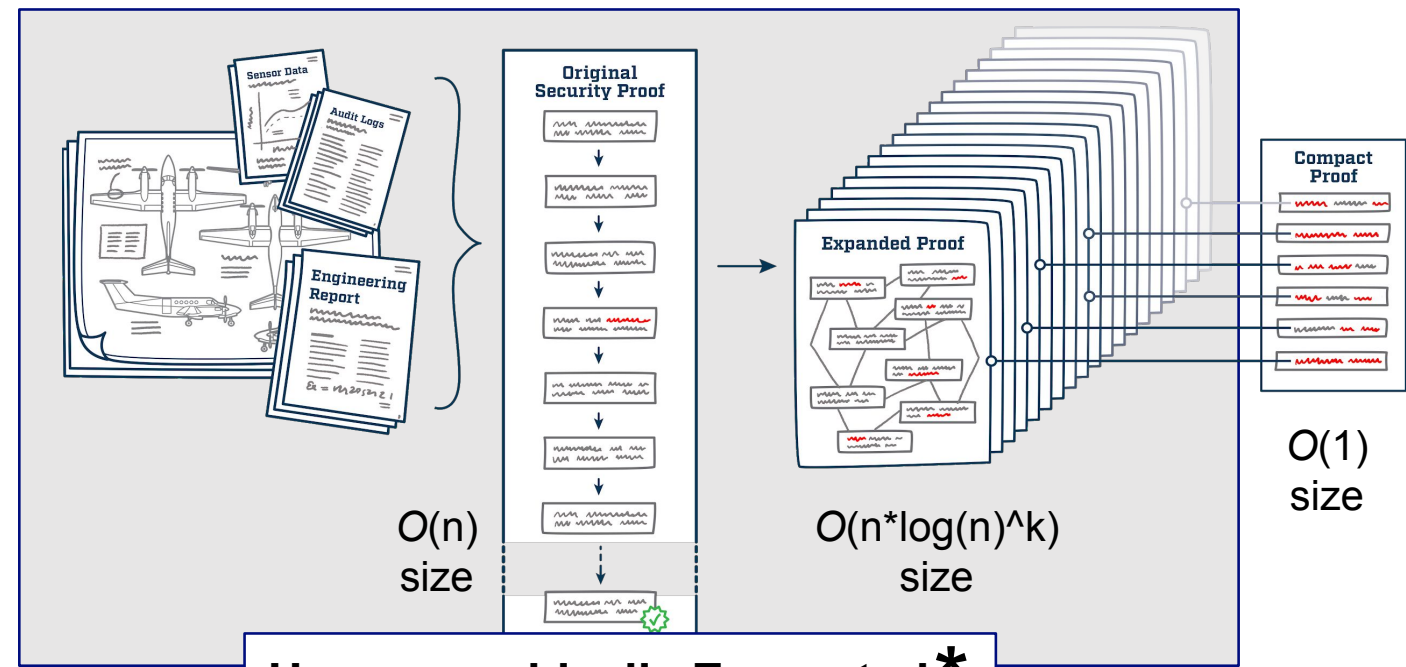

Homomorphically Encrypted * 


\section{zkSNARK Construction for Program Verification [BCGTV13]}

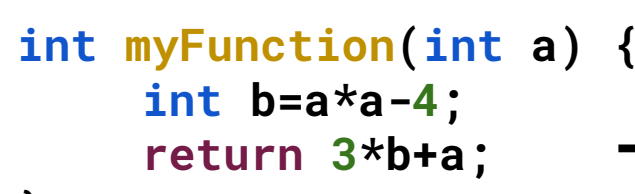

Rank-1 Constraint System (R1CS):
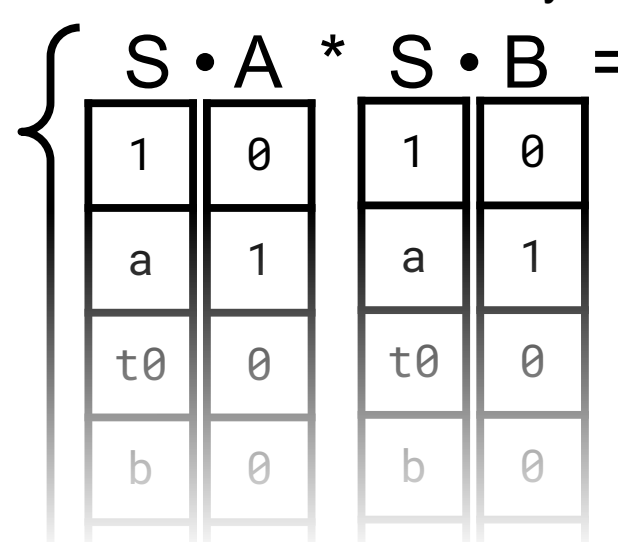

\begin{tabular}{|c|c|}
\hline$S \cdot C$ \\
\hline 1 & 0 \\
\hline$a$ & 1 \\
\hline$t \theta$ & 0 \\
\hline$b$ & 0 \\
\hline
\end{tabular}

\section{libsnark}

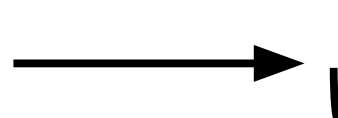

Computation

Arithmetic

Proof Representation

Of Program Execution Circuit

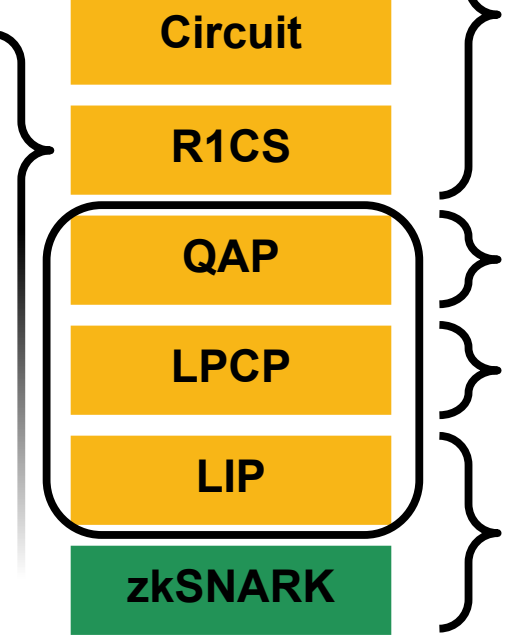

Verifier Binary
Prover Binary
Zero Knowledge Added

Succinctness Added

Interactivity Removed

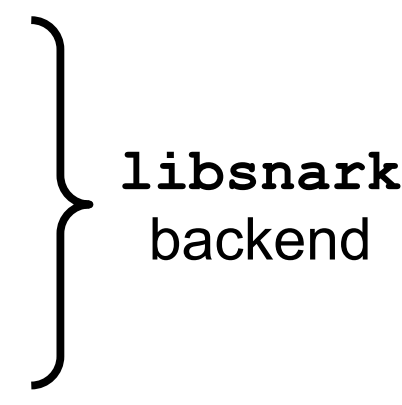




\section{Theory Behind ZKPs (Backup)}




\section{PCPs \& Hardness of Approximation}

\section{Intuition}

Efficient approximation scheme for a problem implies that it an easy to create a good enough looking "fake" solution (witness)

So,

Hard to approximate

$$
\Leftrightarrow
$$

Hard to create a convincing fake witness that appears optimal

$$
\Leftrightarrow
$$

Good witnesses imply that best solutions exist

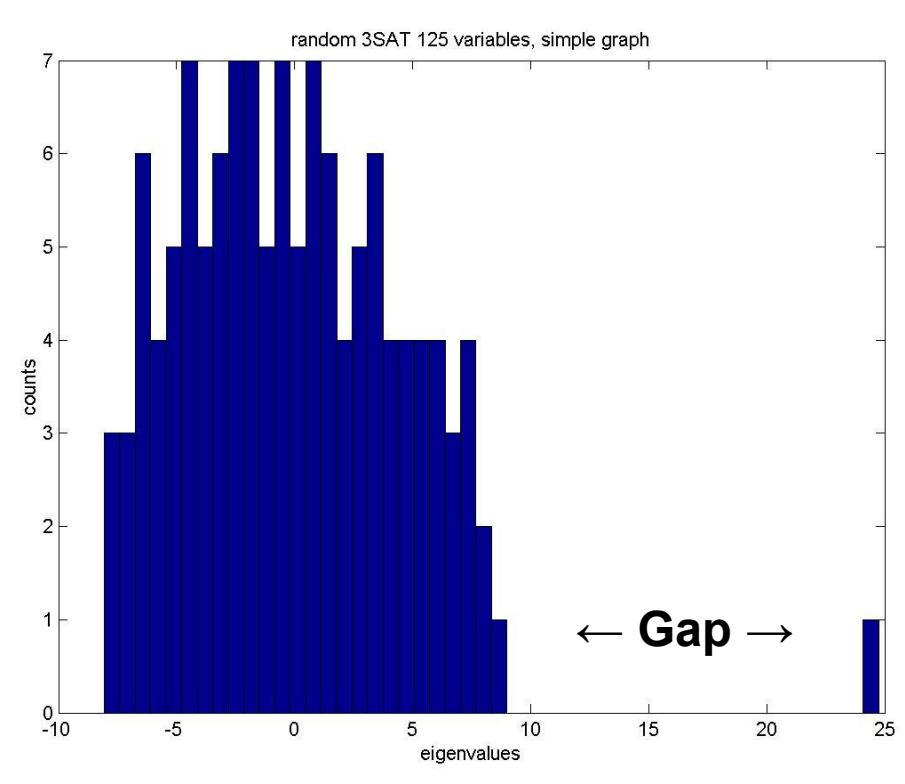

Easy to find

Hard to find 


\section{NIZK Overview}

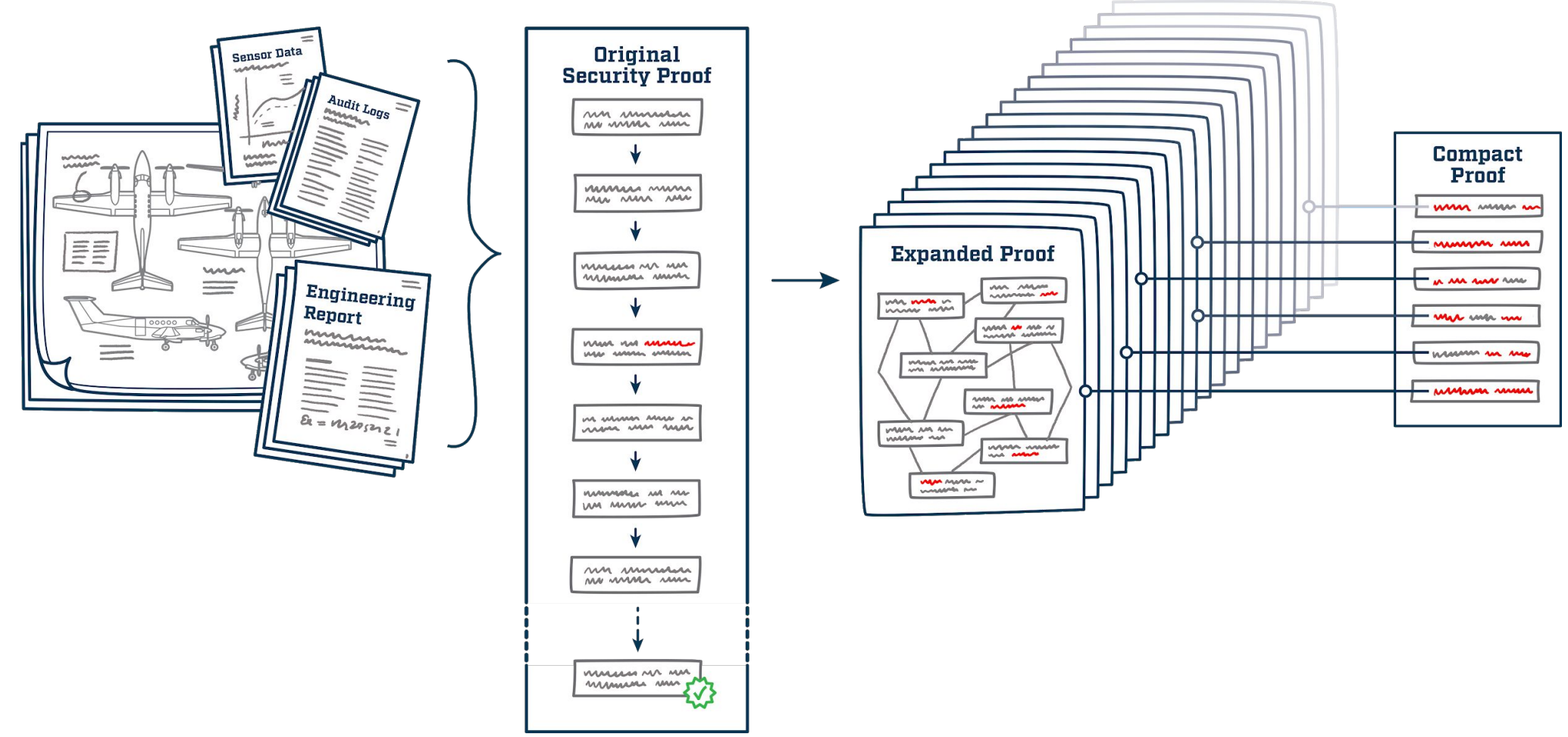




\section{Circuit Evaluation}

1. Publish homomorphically encrypted building blocks for a program

$$
\mathrm{CRS}=\left\{\operatorname{Enc}_{k}(\alpha x), \operatorname{Enc}_{k}\left(\alpha x^{2}\right), \ldots, \operatorname{Enc}_{k}\left(\alpha x^{d}\right)\right\}
$$

2. Prover blindly re-assembles them to compute the desired circuit (e.g. an evaluation of the PCP circuit) and adding random blinds where appropriate

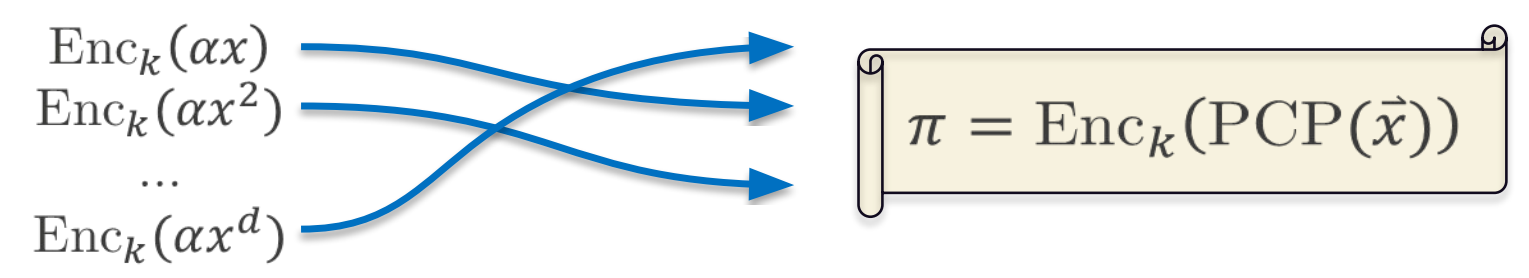

3. Verifier checks content by simply decrypting

$$
\operatorname{Dec}_{k}\left(\operatorname{Enc}_{k}(\operatorname{PCP}(\vec{x}))\right)=\left\{\begin{array}{lr}
1 & \text { if valid } \\
0 & \text { if invalid }
\end{array}\right.
$$

